\title{
Micro Seismic Source Location Based on Teaching Learning- Based Optimization Technique
}

\author{
Prabhat Basnet ${ }^{* 1}$, Wangjin Qiang ${ }^{1}$, Shakil Mahtab ${ }^{2}$ \\ ${ }^{I}$ Civil and Resource Engineering School, University of Science and Technology Beijing, Beijing, China \\ ${ }^{2}$ Department of Geotechnical Engineering, College of Civil Engineering, Tongji University, Shanghai, China
}

*Corresponding Author: Prabhat Basnet, Civil and Resource Engineering School, University of Science and Technology Beijing, Beijing,100083, China, Email:prabhat@ 126.com

\begin{abstract}
In order to overcome the problem caused by the improper tuning of algorithm specific parameters of other heuristic algorithms, the Teaching learning-based optimization (TLBO) technique for solving microseismic $(M S)$ source location with unknown wave velocity has been presented. TLBO is inspired by the teaching and learning process in a classroom and does not require any algorithm-specific parameters and only operates using standard controlling parameters such as population size and the number of iterations. It eliminates the error caused by the improper tuning of algorithmic parameters of a traditionally applied optimization technique in MS source localization and selection of inaccurate velocity model due to the nonhomogeneous structure of the underground structure. The method uses sum of the least squared differences of the observed and calculated arrival time of each sensor as a target function, and TLBO is implemented to calculate the optimal value of source coordinate and unknown velocity. The effectiveness of the TLBO algorithm was verified through the real data obtained from the two different blasting experiments of mine A in China. The result showed that TLBO is easy to compute and does not need any specific tuning of parameters. It also displays fast convergence and better accuracy in calculating MS source location and unknown velocity
\end{abstract}

Keywords: MicroseismicMonitoring, MicroseismicSource Location, Teaching Learning-Based Optimization (TLBO), Unknown Velocity

\section{INTRODUCTION}

Source localization in seismology is very classic and fundamental concerns that provide significance to identify the problems such as seismic activity, Earth's interior structure, acoustic behavior of Rock masses, and rock support under seismic loading related to Rock mechanics and seismology (Codeglia et al., 2017;Warpinski, 2009; H. Wang \& Ge, 2008;Dong et al., 2018). Moreover, source localization plays a significant role in controlling and maintaining the sub-surface risk of earthquake disaster and mining earthquake.

MS Monitoring technology is such a useful tool which have been widely applied in the field of mining and geotechnical engineering to locate the fracturing places in rock masses caused by Rock burst, water inrush, hydraulic fracturing, and various mine hazards (Ge, 2005; Jiang et al., 2019; Ma et al., 2018; A. Wang et al., 2019). MS Monitoring Source localization is a beneficial technique for realtime monitoring of mine seismic hazard because it forecasts the burst location that suggests meaningful prospects in rockburst control (Pu et al., 2019).

However, various factors affect accurate source location such as inaccurate velocity model, improper tuning of optimization algorithm parameters, arrival picking, etc. For these reasons, accurate precision in Source location has become an essential topic of research for decades.

The current phase of source localization in MS monitoring in mining engineering still inspired by the concept of seismology, Geiger established a mathematical approach based on the principle of time International Journal of Mining Science (IJMS)

Page $\mid 46$ 
difference of arrival and calculated the source location of the natural earthquake (Geiger, 1912), based on his theory Lienert et al. established an algorithm called HYPOCENTER (Lienert et al., 1986). However, Nelson and Vidale made a further improvement in the existing algorithm implementing the concept of 3D velocity modeling (Nelson \& Vidale, 1990). Crosson introduced the idea of the Joint inversion localization method, which uses the least square method and inverts a new velocity structure along with source parameters (Crosson, 1976). Pavlis et al. used the parameter separation method to solve the coupled velocity parameters and source parameters separately, which significantly improves the calculation efficiency (Pavlis \& Booker, 1980). Waldhauser and Ellsworth put forward the concept of earthquake location based on the Double difference algorithm (Waldhauser \& Ellsworth, 2000). Relying on such pioneering methods, various scholars have approached new source localization methods, which have been widely applied in mine MS source localization. Lin et al. introduced a combined linear location and Geiger location method that increased the precision of MS source location (Lin et al., 2010). Dong et al. presented a new source location method that does not need to measure the wave velocity in advance (Dong et al., 2011). Chen et al. proposed a Hierarchical strategy based on Particle swarm optimization and much-improved location accuracy (Chen et al., 2009). Huang et al. came with an idea of the relocation method of MS source location that significantly outperformed traditional methods (Huang et al., 2016). Dong et al. introduced an analytical solution for source calculation, which converts the nonlinear equation of time difference of arrival to linear form and obtains the solution (Dong, 2014).

Some of the localization mentioned above methods are based on a linear approach; however, the right seismic problems are more complex and nonlinear. MS source location accuracy is dependent on various factors such as the distribution of sensors array, velocity model, picking of arrival time, which greatly influence the accuracy (Longjun et al. 2013). The current approach is mainly based on nonlinear localization methods, which is far better than the linear approach because the seismic inversion process is carried out by the least square method to find the minimum residual. In this paper, we have proposed a novel Teaching Learning-based optimization (TLBO) technique to find the optimal solution of source location solving a nonlinear equation based on time difference of arrivals. It uses the least-square sum of observed arrival time differences for all pairs of sensors as a target function and solves the source coordinates and wave velocity. Traditionally applied heuristic algorithms in microseismic research often requires proper tuning of algorithm-specific parameters to find the optimal solution; for instance, traditional Evolutionary algorithms such as Genetic algorithm(GA) uses mutation probability, crossover probability, selection operator, etc. and inertia weight, acceleration constants, particle velocity, etc. in case of Swarm intelligence algorithms like Particle swarm optimization (PSO) along with standard controlling parameters such as population size and several generations. Improper tuning of algorithm-specific parameters either increases computational burden or yields the non-unique solution. However, the proposed TLBO overcome such difficulties because it does not require to compute any algorithm-specific parameters; instead, it simply works on relying standard controlling parameters like population size and the number of iterations, which reduces computational burden as well as consumes lots of time.

\section{Mathematical EXPression For SourCe Location Parameters}

The $\mathrm{n}$ number of microseismic sensors are placed in a monitoring area with known three-dimensional coordinates which are $\left(x_{1}, y_{1}, z_{1}\right),\left(x_{2}, y_{2}, z_{2}\right), \ldots . .\left(x_{i}, y_{i}, z_{i}\right)$ and $(\mathrm{i}=1,2,3 \ldots . \mathrm{n})$. Most Microseismic phases are body waves; therefore, once a microseismic event occurs, energy emits in the form of seismic waves called $\mathrm{P}$-waves, which propagates from the source location $\left(x_{0}, y_{0}, z_{0}\right)$ to each sensor and the arrival time of $\mathrm{P}$-wave to the $\mathrm{i}^{\text {th }}$ sensor is recorded as $t_{i}$. If we consider propagation media as a homogeneous medium, then the equivalent average propagation velocity of the $\mathrm{P}$-wave in the medium is V. The general principle of MS source location is shown in (Fig.1).

International Journal of Mining Science (IJMS)

Page $\mid 47$ 


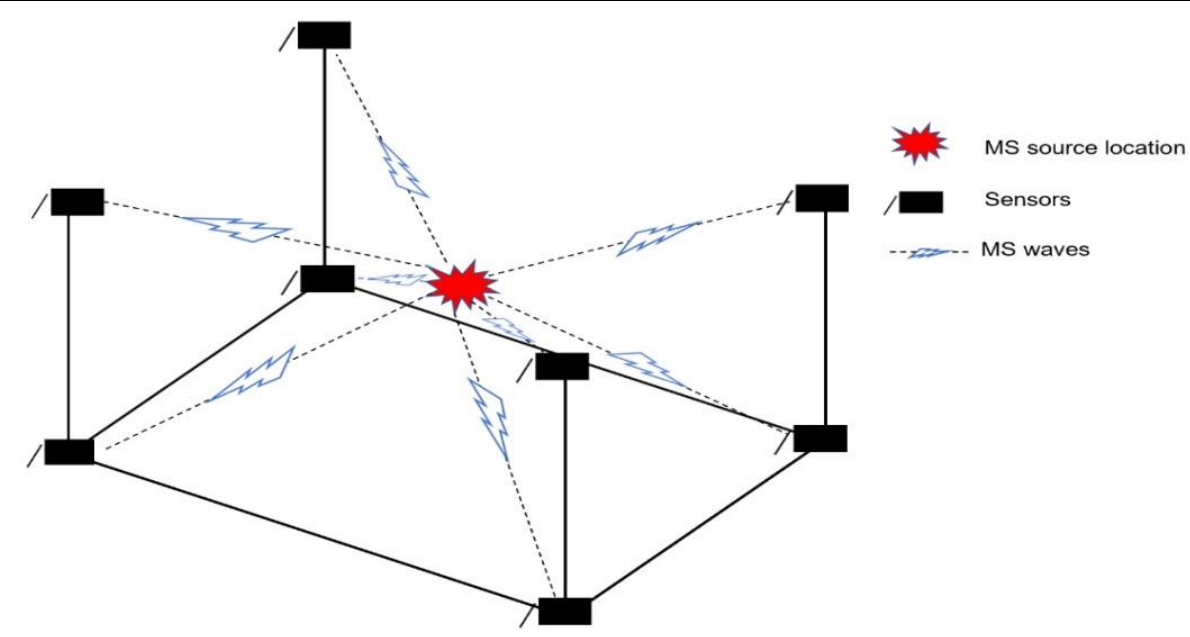

Fig1. Schematic diagram of MS source location principle

The distance between the MS source and the given sensor can be expressed as:

$R_{i}=\sqrt{\left(x_{0}-x_{i}\right)^{2}+\left(y_{0}-y_{i}\right)^{2}+\left(z_{0}-z_{i}\right)^{2}}$

According to the distance formula in terms of velocity and time, $R_{i}$ can be written as:

$R_{i}=\mathrm{V} t_{i}$

From equation (1) and (2), we can obtain following equation,

$t_{i}=\frac{\sqrt{\left(x_{0}-x_{i}\right)^{2}+\left(y_{0}-y_{i}\right)^{2}+\left(z_{0}-z_{i}\right)^{2}}}{V}$

According to the principle of time difference of arrival for the two different sensors, $i$ and $j$ equation (3) can be expressed as:

$\Delta \mathrm{t}_{i j}=t_{i}-t_{j}=\frac{R_{i-R_{j}}}{V},(\mathrm{i}, \mathrm{j}=1,2 \ldots \ldots \mathrm{n})=\mathrm{f}\left(x_{i}, y_{i}, z_{i}, x_{j}, y_{j}, z_{j}, x_{0}, y_{0}, z_{0}, V\right)$

In equation (4) sensor coordinates $\left(x_{i}, y_{i}, z_{i}\right),\left(x_{j}, y_{j}, z_{j}\right)$ and arrival time for two different sensors $t_{i}, t_{j}$ are already known parameters, where Microseismic source coordinates $x_{0}, y_{0}, z_{0}$ Moreover, the equivalent average velocity $\mathrm{V}$ are unknown parameters. Therefore, as it is a three-dimensional source location technique that requires more than four sensors to solve unknown parameters solving the nonlinear equations.

For observed data $\left(x_{i}, y_{i}, z_{i}, x_{j}, y_{j}, z_{j}\right)$ we can establish a regression value $\Delta \hat{t}_{i j}$. The difference between $\Delta \mathrm{t}_{i j}$ and $\Delta \hat{t}_{i j}$ shows the degree of variation between observed data and calculated (regression) data. The sum of their squared deviations reflects how well data fits the line. The more the deviation between observed and calculated data becomes small, the more the data fits the line. The equation for the estimation of the MS source location can be expressed as:

$\mathrm{f}\left(x_{0}, y_{0}, z_{0}, V\right)=\sum_{i, j=1}^{n}\left[\Delta \hat{t}_{i j}-\frac{R_{i-} R_{j}}{V}\right]^{2}=\min$

Equation (5) is a nonnegative function of unknown variables $\left(x_{0}, y_{0}, z_{0}, V\right)$, which always has the minimum and the calculated microseismic source, and equivalent average velocity reaches the global minimum. The benefit of the above method is that it is not necessary to measure the average velocity in advance, and there is no need to fit the original time of the event beforehand.

In order to calculate the origin time $t_{0}$ using time difference location principle equation can be described below:

$\min _{t_{0}} Q\left(t_{0}\right)=\min _{t_{0}} \quad \sum_{i=1}^{n}\left[t_{i}-t_{0}-\frac{R_{i}\left(\hat{x}_{0}, \hat{y}_{0}, \hat{z}_{0}\right)}{\widehat{V}}\right]^{2}$

Where, $\hat{x}_{0}, \hat{y}_{0}, \hat{z}_{0}$ and $\widehat{V}$ are calculated source location and equivalent average velocity. If we consider the propagation medium between source and sensors is homogeneous to $\min _{t_{0}} Q\left(t_{0}\right) \approx 0$, origin time can be obtained as below:

International Journal of Mining Science (IJMS)

Page $\mid 48$ 
$\hat{t}_{0} \approx \frac{1}{n} \sum_{i=1}^{n}\left[t_{i}-\frac{R_{i}\left(\hat{x}_{0}, \hat{y}_{0}, \hat{z}_{0}\right)}{\widehat{V}}\right]^{2}$

To get the solution of origin time, the regression value $\hat{x}_{0}, \hat{y}_{0}, \hat{z}_{0}$ and $\widehat{V}$ should be calculated first using Equation (5), then after calculated value can be substituted in Equation (7) to get the origin time.

As we know, Equation (5) is a nonnegative function, and the global minimum always exists, which can be found by the nonlinear fitting methods.

As there are two broad groups of population-based heuristic algorithms to solve the nonlinear problems, evolutionary algorithms (EA) and swarm intelligence (SI) based algorithms hence all these probabilistic algorithms are operated under standard controlling parameters such as Population size, the total number of generations, select size and so on. Besides having controlling parameters, various algorithms have their algorithm-specific control parameters, which is a very crucial factor that directly affects the performance of the algorithm. Therefore, it is not so easy to operate these algorithms as one should have proper knowledge of parameter tuning, and problems such as the inappropriate selection of parameters sometime might lead to getting nonunique solution and solution can be easily trapped into local minima. In order to overcome such problems in solving nonlinear MS source location, we have introduced a Teaching Learning-based optimization technique which only requires common controlling parameters like population size and iteration number to be operated, this algorithm is simple to use, and there is less fear of trapping on local minima because it converges monotonically due to the method of greedy selection in each iteration.

\section{Tlbo For Solving Source Location Problem}

\subsection{Working Principle of TLBO Algorithm}

TLBO algorithm is an optimization technique that simply works with population size and some generations. It is developed by Rao et al. (2011), which uses a population of the solution in order to proceed with a global solution, and it is mainly inspired by the teaching and learning process in a classroom (Rao, Savsani, \& Vakharia, 2011, 2012). In the TLBO, a set of learners is considered as the population of solutions; different design variables will be similar to various subjects offered, learners and the fitness of the solutions are considered as results or grades. The algorithm updates the results of each learner of the class by learning from the teacher and learning from the interaction between other learners or partners. The Working process of TLBO mainly relies on two operations: 'Teacher phase' and 'learner phase.'

\subsubsection{Teacher Phase}

In the teacher phase, learners acquire knowledge from teachers, depending upon the learner's capability of learning, the teacher attempts to increase the mean result of the class in the subject taught by him/her. If it is assumed at any iteration i, there are ' $\mathrm{m}$ ' numbers of subjects (designed variables), ' $\mathrm{n}$ ' number of learners (population size, $\mathrm{k}=1,2 \ldots \mathrm{n}$ ), then $M_{j, i}$ is the mean result of learners in particular subject ' $\mathrm{j}$ ' $(\mathrm{j}=1,2 \ldots \mathrm{m})$. The teacher is often considered as the highly knowledgeable person who trains the learner to gain outstanding result; therefore, the learner with the best fitness identified by the algorithm is the teacher, and the updated solution is calculated by taking the difference between the existing mean result of each subject concerning the result of the teacher for each subject. The expression is given by,

Difference_mean ${ }_{j, k, i}=r_{i}\left(X_{j, k b e s t, i}-T_{F} M_{j, i}\right)$

Where, $X_{j, k b e s t, i}$ is the learner with the best fitness (Teacher). $r_{i}$ is the random number whose value lies in between $[0,1], T_{F}$ are a teaching factor and its value heuristically set to be either 1 or $2 . T_{F}$ is not a parameter of algorithm and do not need to give any input; its value is randomly selected by an algorithm using the expression below,

$T_{F}=$ round $[1+\operatorname{rand}(0,1)]$

Based on Equation (8) the position of each learner is updated, which is given by:

$X_{j . k, i}^{\prime}=X_{j, k, i}+$ Difference_mean ${ }_{j, k, i}$ 
Where, $X_{j . k, i}^{\prime}$ is the updated value of $X_{j, k, i}$. If the updated new value $X_{j . k, i}^{\prime}$ is better than the old value $X_{j, k, i}$ The only new value is accepted; otherwise, the value remains unchanged; whatever solution comes from the teacher phase will be input for the learner phase.

\subsubsection{Learner Phase}

This is the second part of the algorithm where learners try to increase or reinforce their knowledge selecting learning partners through various ways such as interacting, communicating, and discussing with each other who has more knowledge than him/her. Randomly select two learning partners, say, $Y$ and Z, such that $X_{\text {total }-Y, i}^{\prime} \neq X_{\text {total }-Z, i}^{\prime}$, where

$X_{\text {total }-Y, i}^{\prime}$ and $X_{\text {total }-Z, i}^{\prime}$ are the updated function values of $X_{\text {total }-Y, i}$ and $X_{\text {total }-z, i}$ of learning partners $Y$ and $Z$ that are obtained at the end of the Teacher phase.

$X_{j, Y, i}^{\prime \prime}=X_{j, Y, i}^{\prime}+r_{i}\left(X_{j, Y, i}^{\prime}-X_{j, Z, i}^{\prime}\right)$, In case of $X_{\text {total }-Y, i}^{\prime}<X_{\text {total }-Z, i}^{\prime}$

$X_{j, Y, i}^{\prime \prime}=X_{j, Y, i}^{\prime}-r_{i}\left(X_{j, Y, i}^{\prime}-X_{j, Z, i}^{\prime}\right)$, In case of $X_{\text {total }-Y, i}^{\prime}>X_{\text {total }-Z, i}^{\prime}$

Similarly, like in the Teacher phase $X_{j, Y, i}^{\prime \prime}$ is accepted only if it has a better function value. Equation (11) and (12) are only applicable to a minimization problem. However, for maximization problem

Equation (13) and (14) should be preferred.

$X_{j, Y, i}^{\prime \prime}=X_{j, Y, i}^{\prime}+r_{i}\left(X_{j, Y, i}^{\prime}-X_{j, Z, i}^{\prime}\right)$, In case of $X_{\text {total }-Y, i}^{\prime}>X_{\text {total }-Z, i}^{\prime}$

$X_{j, Y, i}^{\prime \prime}=X_{j, Y, i}^{\prime}-r_{i}\left(X_{j, Y, i}^{\prime}-X_{j, Z, i}^{\prime}\right)$, In case of $X_{\text {total }-Y, i}^{\prime}<X_{\text {total }-Z, i}^{\prime}$

In this way, the algorithm is completed.

\subsection{TLBO for Optimization of Source Location}

Equation (5) is a multiple local extremum nonlinear optimization problem; the TLBO is mainly designed for optimization of such problems and can be implemented in order to search optimal value of three-dimensional microseismic source location and propagation velocity i.e.

$\left(x_{0}, y_{0}, z_{0}, \mathrm{~V}\right)$.

The procedure that is involved is described below.

Step 1. Initializethe model parameters of MS source location and TLBO parameters,

TLBO parameters mainly include population size and the number of iterations.

Step 2. Start Teacher phase to calculate the mean of the population of designed variables and select the best solution(teacher) using Eq. (5)

Step 3. Update the solution using Eq. (10); if a newly obtained solution is better than the old one, accept the new one; otherwise, keep the previous solution. In this way, the Teacher phase ends.

Step 4. Start the Learner phase randomly selecting two learners, say Y and Z, check the better function value, if the function value $X_{\text {total }-Y, i}^{\prime}<X_{\text {total }-Z, i}^{\prime}$ prefer Equation (11) otherwise Equation (12)

Step 5. Upgrade the solution using Equation (11) in case of $X_{\text {total }-Y, i}^{\prime}<X_{\text {total }-Z, i}^{\prime}$ otherwise, use Equation (12); if the upgraded solution outperforms the old solution, then take a new solution if not keep the old solution.

Step 6. If the condition satisfied, obtain the final solution $\left(x_{0}, y_{0}, z_{0}, \mathrm{~V}\right)$, if not go back to Step 1.

Step 7. After obtaining the source coordinates, and equivalent velocity, terminate the algorithm.

The flow chart of TLBO to calculate source location is shown in (Fig.2). 


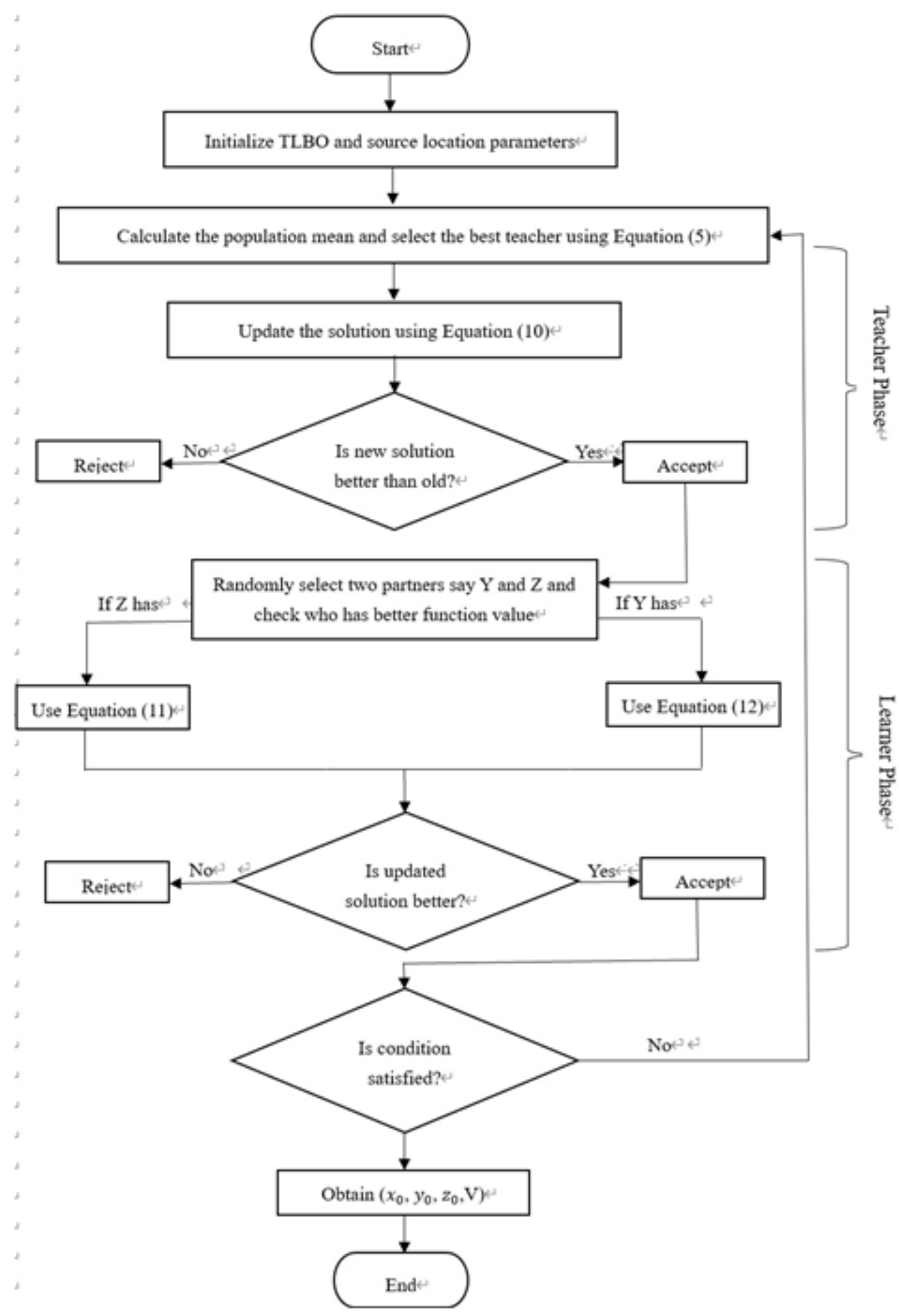

Fig2. Flow chart of microseismic source location algorithm based on TLBO

\subsection{Discussion of TLBO Parameters}

TLBO is based on common controlling parameters like population size and a number of generations or maximum iterations; therefore, TLBO does not require any specific parameters like other traditional heuristic algorithms, which along with common controlling parameters, also require algorithm-specific parameters to be defined before operated. Hence, in TLBO, we do not have to deal with other parameters except common controlling parameters, which saves much time and makes computation more convenient.

\section{Case Study}

From the underground Geological condition, mine A in central China is a typical mine that is seriously threatened by rock bursts. A multi-channel MS monitoring system of ARAMIS M/E was installed to monitor the rock fracturing process; eight geophones were installed in 2613 working face of mine to detect the seismic events as shown in (Fig.3). 


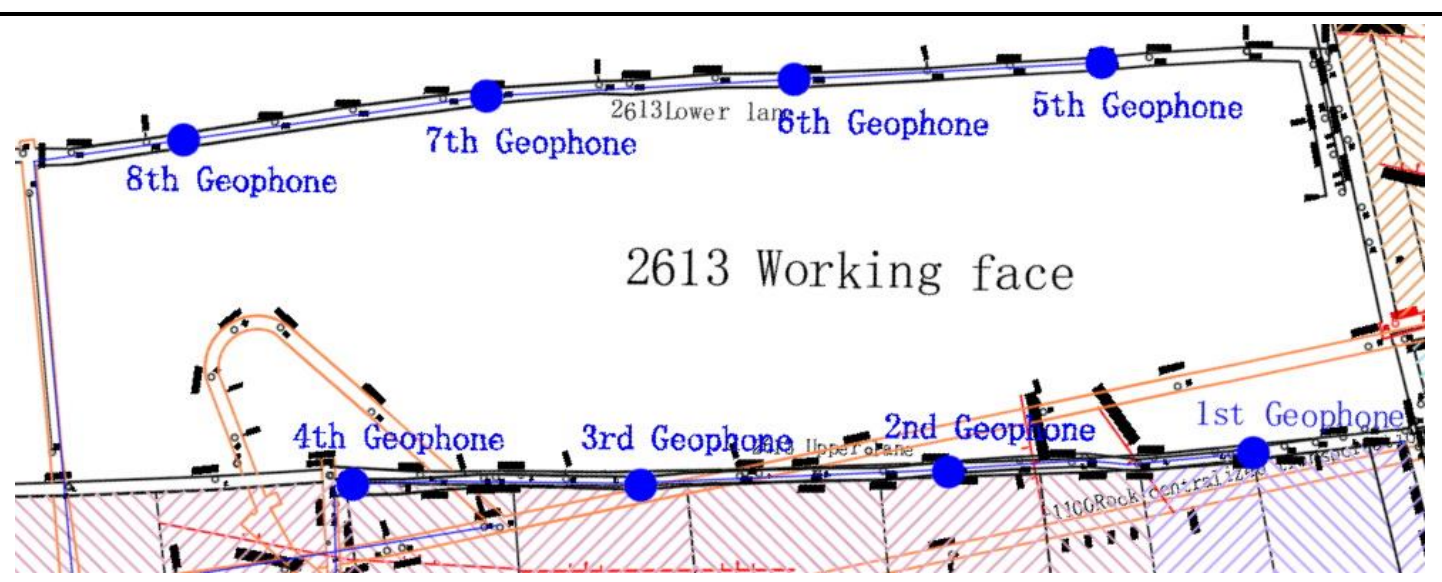

Fig3. Layout of microseismic monitoring system in 2613 working face

Two different blasting experiments were held with known positions to verify the validity of the TLBO algorithm. During the first experiment which was conducted on 2019-08-08between the $1^{\text {st }}$ and $2^{\text {nd }}$ geophones all devices detected the microseismic signals (i.e. all 8 geophones), similarly, on 2019-0809 second blasting experiment was conducted between $7^{\text {th }}$ and $8^{\text {th }}$ geophones where all of the geophones received the signals. The received data were preprocessed in order to get good quality signal. The position coordinates of first and second blasting experiments are $(20513933.5,3973994.8$, $-1088.34)$ and $(20513882.35,3974172.25,-1174.23)$ respectively.

The coordinates of 8 geophones and the time of arrival to each sensor is shown in Table (1).

The schematic diagram of source position and sensors position is shown in (Fig.4)

Table1. Coordinates of sensors and arrival time from burst point

\begin{tabular}{|c|c|c|c|c|c|}
\hline \multirow{2}{*}{$\begin{array}{l}\text { Geophone } \\
\text { no. }\end{array}$} & \multicolumn{3}{|c|}{ Coordinates of Geophones $(\mathrm{m})$} & \multicolumn{2}{|l|}{ Arrival time(S) } \\
\hline & $\mathrm{x}$ & $\mathrm{y}$ & $\mathrm{z}$ & First experiment & $\begin{array}{l}\text { Second } \\
\text { experiment }\end{array}$ \\
\hline \multirow{8}{*}{$\begin{array}{l}1 . \\
2 . \\
3 \\
4 . \\
5 . \\
6 . \\
7 . \\
8 .\end{array}$} & 20513973.1 & 3973934.6 & -1076.6 & 0.0205 & 0.0774 \\
\hline & 20513903.9 & 3973995.5 & -1080.1 & 0.0094 & 0.0599 \\
\hline & 20513836.5 & 3974060.6 & -1089.9 & 0.0336 & 0.0410 \\
\hline & 20513777 & 3974117 & -1095.8 & 0.0566 & 0.0391 \\
\hline & 20514038.6 & 3974046.2 & -1165.8 & 0.0389 & 0.0579 \\
\hline & 20513969.4 & 3974107.1 & -1170.7 & 0.0401 & 0.0319 \\
\hline & 20513903.4 & 3974168.2 & -1174.2 & 0.0551 & 0.0078 \\
\hline & 20513842.8 & 3974215.3 & -1174.8 & 0.0717 & 0.0159 \\
\hline
\end{tabular}

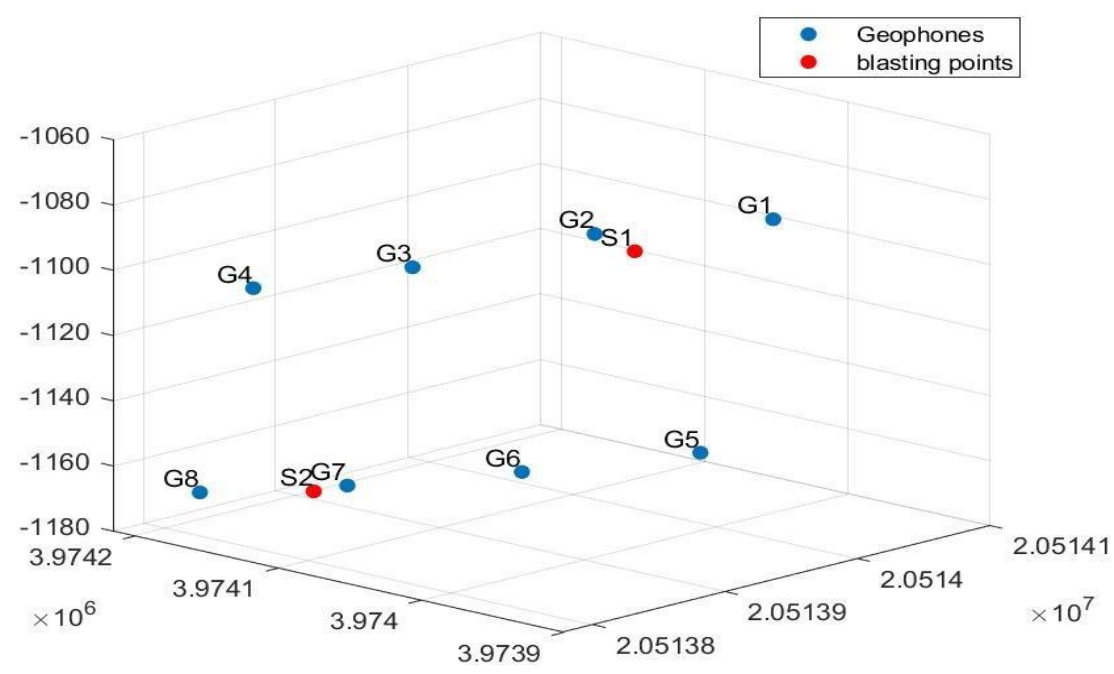

Fig4. Schematic diagram of relative positions of Geophones and blasting points

The blasting experiment was carried out on the 2613 working face where the first experiment was done in between $1^{\text {st }}$ and $2^{\text {nd }}$ geophones similarly, the second experiment was done in between $7^{\text {th }}$ and $8^{\text {th }}$ geophones, the depth of the borehole is $10 \mathrm{~m}$, and the borehole diameter is $42 \mathrm{~mm}$, amount of explosive used is $1 \mathrm{~kg}$,the sealing length of borehole is not less than half of the hole length. 
The blasting is done undermining personnel; after the experiment, the authentic calculated propagation velocity in the rock media is approximately $3533 \mathrm{~m} / \mathrm{s}$. However, due to the heterogeneous properties of rock, it is always hard to get the accurate velocity; and finally, the TLBO algorithm was used to solve the microseismic source location and equivalent velocity; the parameters for the TLBO algorithm are as follows: population size is 500 , total number iteration $T_{\max }=3000$. The teaching factor $T_{F}$ is calculated using Equation (9), and random number $\mathrm{r}$ is decided by the algorithm. Based on the observed data in Table 1.The TLBO was implemented on MATLAB to obtain the results, and the results showed that TLBO achieved high performance in calculating MS source location and unknown velocity value, the obtained result for both the experiment was calculated by executing the algorithm for 20 times and taking the average value for each experiment which is shown in Table (2).

Table2. Error comparison of TLBO for first and second experiment

\begin{tabular}{|c|c|c|c|c|c|}
\hline & & $\Delta x_{0}$ & $\Delta y_{0}$ & $\Delta z_{0}$ & $\Delta \mathrm{V}$ \\
\hline First experiment & TLBO & 2.58 & 1.03 & -0.90 & -2.41 \\
\hline Second experiment & TLBO & -4.97 & 0.93 & 0.57 & 2.73 \\
\hline Absolute average error & & 3.77 & 0.98 & 0.73 & 2.57 \\
\hline
\end{tabular}

From the result, we can conclude that the result calculated by TLBO is better due to its robustness that requires less parameter tuning. The TLBO algorithm can automatically approach the true values with the given initial parameter range, and the absolute average error for both experiments together using TLBO in X, Y Z axis, and velocity is $3.77,0.98,0.73 \mathrm{~m}$, and $2.57 \mathrm{~m} / \mathrm{s}$ respectively, which represents the errors are below $4 \%$. Therefore, TLBO can achieve good performance accuracy in solving source location. From the case studies, it demonstrates that the TLBO algorithm is better in solving microseismic source location and equivalent average velocity than traditional optimization algorithm because it avoids the burden of tuning lots of algorithm specific parameters which sometimes yields non unique result. The algorithm almost took 2.30 minutes to solve the optimal solution and has a very fast convergence speed and reaches global optima quickly. It is easy to set initial parameters because TLBO is more accurate in fitting the nonlinear relationship of each signal receiving device and time difference, which significantly reduces the impact of the velocity error in source localization. The absolute average error in $\mathrm{x}, \mathrm{y}$, and $\mathrm{z}$-direction along with wave velocity calculated using TLBO is shown in (Fig.5).

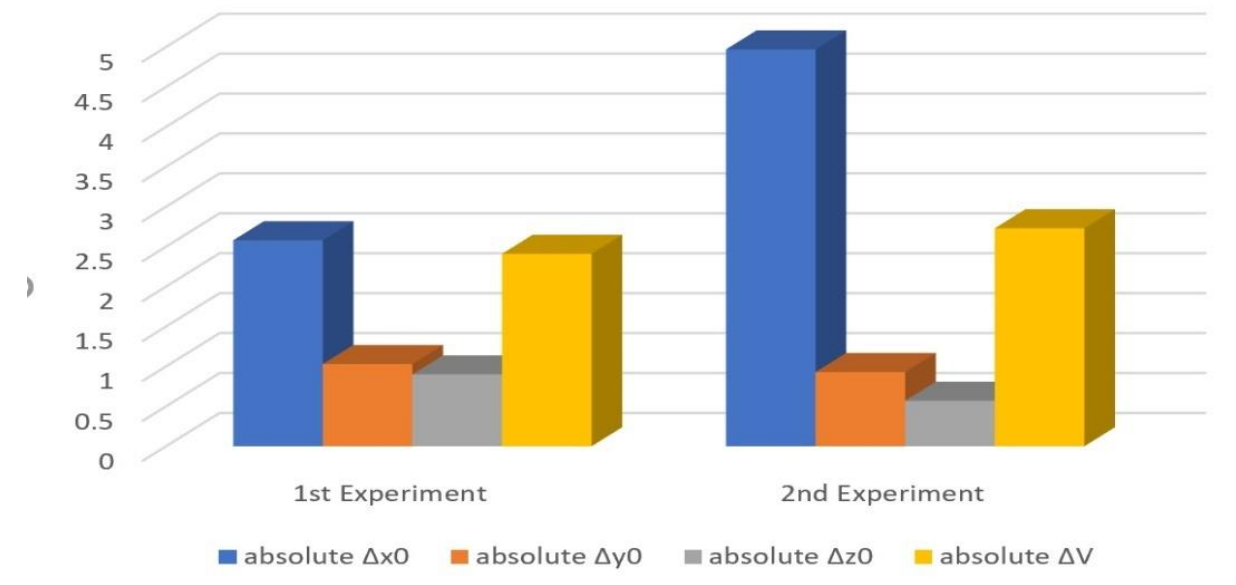

\section{CONCLUSION}

Fig5. The absolute error for $1^{\text {st }}$ and $2^{\text {nd }}$ experiment

Microseismic source location is always influenced by factors such as premeasured wave velocity and the improper tuning of the algorithm-specific parameters of heuristic algorithms. To overcome such problems TLBO technique is proposed to solve the microseismic source location with an undetermined velocity model. TLBO is simple and easy to execute as it only requires population size and the iteration number as an algorithm parameter and finds the optimal solution quickly. The method takes the sum of least squares difference of observed and calculated value as a target function to solve the source coordinates and unknown equivalent wave velocity. The efficiency of the proposed method was verified by the two real field blasting experiment data from mine A in China. The obtained final result depicts algorithm perform fast computation speed as well as approaches true solution without tuning lots of parameters compared with traditional optimization algorithm. 
Comparative analysis shows that TLBO performs better in solving positioning accuracy and wave velocity. The benefit of TLBO is that it does not need any specific knowledge for parameter tuning like in traditionally applied heuristic algorithms, which prevents from yielding non-unique solution.

\section{REFERENCES}

[1] Chen, B., Feng, X.-T., Li, S., Yuan, J., \& Xu, S. (2009). Microseism source location with hierarchical strategy based on particle swarm optimization. Yanshilixue Yu Gongcheng Xuebao/Chinese Journal of Rock Mechanics and Engineering, 28, 740-749.

[2] Codeglia, D., Dixon, N., Fowmes, G. J., \& Marcato, G. (2017). Analysis of acoustic emission patterns for monitoring of rock slope deformation mechanisms. Engineering Geology, 219, 21-31. doi: https://doi.org/10.1016/j.enggeo.2016.11.021

[3] Crosson, R. S. (1976). Crustal structure modeling of earthquake data: 1. Simultaneous least squares estimation of hypocenter and velocity parameters. 81(17), 3036-3046. doi:10.1029/JB081i017p03036

[4] Dong, L. (2014). An Analytical Solution for Acoustic Emission Source Location for Known P Wave Velocity System. Mathematical Problems in Engineering, 2014, 1-6.

[5] Dong, L., Li, X., Tang, L., \& Gong, F. (2011). Mathematical functions and parameters for microseismic source location without pre-measuring speed. Yanshilixue Yu Gongcheng Xuebao/Chinese Journal of Rock Mechanics and Engineering, 30, 2057-2067.

[6] Dong, L., Sun, D., Li, X., Ma, J., Zhang, L., \& Tong, X. (2018). Interval non-probabilistic reliability of surrounding jointed rockmass considering microseismic loads in mining tunnels. Tunnelling and Underground Space Technology, 81, 326-335. doi:https://doi.org/10.1016/j.tust.2018.06.034

[7] Ge, M. (2005). Efficient mine microseismic monitoring. International Journal of Coal Geology, 64(1), 4456. doi:https://doi.org/10.1016/j.coal.2005.03.004

[8] Geiger, L. (1912). Probability method for determination of earthquake epicenters from arrival time only. Bull. St. Louis Univ., 8, 60-71.

[9] Huang, L.-q., Li, X.-b., Dong, L.-j., Zhang, C.-X., \& Liu, D. (2016). Relocation method of microseismic source in deep mines. Transactions of Nonferrous Metals Society of China, 26(11), 2988-2996. doi:https://doi.org/10.1016/S1003-6326(16)64429-1

[10] Jiang, Z., Li, Q., Hu, Q., Chen, J., Li, X., Wang, X., \& Xu, Y. (2019). Underground microseismic monitoring of a hydraulic fracturing operation for CBM reservoirs in a coal mine. Energy Science \& Engineering, 7. doi:10.1002/ese3.329

[11] Lienert, B., Berg, E., \& Frazer, N. (1986). HYPOCENTER: An earthquake location method using centred, scaled and adaptively damped least squares. Bulletin of the Seismological Society of America, 76, 771783.

[12] Lin, F., Li, S., Xue, Y., Xu, H. J. C. J. o. R. M., \& Engineering. (2010). MICROSEISMIC SOURCES LOCATION METHODS BASED ON DIFFERENT INITIAL VALUES. 29(5), 996-1002.

[13] Longjun, D., Xibing, L. I. J. e., \& Review, T. (2013). Main Influencing Factors for the Accuracy of Microseismic Source Location.

[14] Ma, T.-H., Tang, C.-A., Tang, S.-B., Kuang, L., Yu, Q., Kong, D.-Q., \& Zhu, X. (2018). Rockburst mechanism and prediction based on microseismic monitoring. International Journal of Rock Mechanics and Mining Sciences, 110, 177-188. doi:https://doi.org/10.1016/j.ijrmms.2018.07.016

[15] Nelson, D., \& Vidale, E. J. B. o. t. S. S. o. A. (1990). Earthquake locations by 3-D finite-difference travel times. 80(2), 395-410.

[16] Pavlis, G. L., \& Booker, J. R. (1980). The mixed discrete-continuous inverse problem: Application to the simultaneous determination of earthquake hypocenters and velocity structure. 85(B9), 4801-4810. doi:10.1029/JB085iB09p04801

[17] Pu, Y., Apel, D. B., Liu, V., \& Mitri, H. (2019). Machine learning methods for rockburst prediction-stateof-the-art review. International Journal of Mining Science and Technology, 29(4), 565-570. doi:https://doi.org/10.1016/j.ijmst.2019.06.009

[18] Rao, R. V., Savsani, V. J., \& Vakharia, D. P. (2011). Teaching-learning-based optimization: A novel method for constrained mechanical design optimization problems. Computer-Aided Design, 43(3), 303315. doi:https://doi.org/10.1016/j.cad.2010.12.015

[19] Rao, R. V., Savsani, V. J., \& Vakharia, D. P. (2012). Teaching-Learning-Based Optimization: An optimization method for continuous non-linear large scale problems. Information Sciences, 183(1), 1-15. doi:https://doi.org/10.1016/j.ins.2011.08.006

[20] Waldhauser, F., \& Ellsworth, W. L. (2000). A Double-Difference Earthquake Location Algorithm: Method and Application to the Northern Hayward Fault, California. Bulletin of the Seismological Society of America, 90(6), 1353-1368. doi:10.1785/0120000006 \% J Bulletin of the Seismological Society of America 
[21] Wang, A., Song, D., He, X., Dou, L., Li, Z., Zu, Z., . . Zhao, Y. (2019). Investigation of coal and gas outburst risk by microseismic monitoring. PloS one, 14(5), e0216464-e0216464. doi:10.1371/journal. pone. 0216464

[22] Wang, H., \& Ge, M. (2008). Acoustic emission/microseismic source location analysis for a limestone mine exhibiting high horizontal stresses. International Journal of Rock Mechanics and Mining Sciences, 45(5), 720-728. doi:https://doi.org/10.1016/j.ijrmms.2007.08.009

[23] Warpinski, N. (2009). Microseismic Monitoring: Inside and Out. Journal of Petroleum Technology, 61(11), 80-85. doi:10.2118/118537-JPT

Citation: Prabhat Basnet, (2020)" Micro Seismic Source Location Based on Teaching Learning-Based Optimization Technique”, International Journal of Mining Science (IJMS), 6(3), pp. 46-55. DOI: http://doi.org/10.20431/24549460.0603005

Copyright: () 2020 Prabhat Basnet, This is an open-access article distributed under the terms of the Creative Commons Attribution License, which permits unrestricted use, distribution, and reproduction in any medium, provided the original author and source are credited. 